\title{
Queensland diabetic foot hospitalisations (2005-10): in what state is our foot hospital problem?
}

\author{
Peter A Lazzarini ${ }^{1,2,3^{*}}$, Sharon R O'Rourke ${ }^{4}$, Anthony W Russell ${ }^{5,6}$, Patrick H Derhy ${ }^{7}$, Maarten C Kamp ${ }^{8,9}$ \\ From Australasian Podiatry Council Conference 2013 \\ Sydney, Australia. 2-5 June 2013
}

\section{Background}

Diabetes foot complications are a leading cause of overall avoidable hospital admissions. Since 2006, the Queensland Diabetes Clinical Network has implemented programs aimed at reducing diabetes-related hospitalisation. The aim of this retrospective observational study was to determine the incidence of diabetes foot-related hospital admissions in Queensland from 2005 to 2010.

\section{Methods}

Data on all primary diabetes foot-related admissions in Queensland from 2005-2010 was obtained using diabetes foot-related ICD-10-AM (hospital discharge) codes. Queensland diabetes foot-related admission incidences were calculated using general population data from the Australian Bureau of Statistics. Furthermore, diabetes foot-related sub-group admissions were analysed. Chi-squared tests were used to assess changes in admissions over time.

\section{Results}

Overall, 24,917 diabetes foot-related admissions occurred, resulting in the use of 260,085 bed days or $1.4 \%$ of all available Queensland hospital bed days $(18,352,152)$. The primary reasons for these admissions were foot ulcers $(49.8 \%)$, cellulitis $(20.7 \%)$, peripheral vascular disease (17.8\%) and osteomyelitis (3.8\%). The diabetes foot-related admission incidence among the general population (per 100,000) reduced by $22 \%(103.0$ in 2005 , to 80.7 in 2010, $p<0.001$ ); bed days decreased by $18 \%(1,099$ to $904, p<0.001)$.

\section{Conclusion}

Diabetes foot complications appear to be the primary reason for 1.4 out of every 100 hospital beds used in Queensland. There has been a significant reduction in the incidence of diabetes foot-related admissions in Queensland between 2005 and 2010. This decrease has coincided with a corresponding decrease in amputations and the implementation of several diabetes foot clinical programs throughout Queensland.

\section{Author details}

${ }^{1}$ Allied Health Research Collaborative, Metro North Hospital \& Health Service, Queensland Health, Brisbane, Queensland, 4032, Australia. ${ }^{2}$ Department of Podiatry, Metro North Hospital \& Health Service, Queensland Health, Brisbane, Queensland, 4032, Australia. ${ }^{3}$ School of Clinical Sciences, Queensland University of Technology, Brisbane, Queensland, 4059, Australia. ${ }^{4}$ Cairns Diabetes Centre, Queensland Health, Cairns, Queensland, 4870, Australia. ${ }^{5}$ Department of Diabetes \& Endocrinology, Princess Alexandra Hospital, Brisbane, Queensland, 4012, Australia. ${ }^{6}$ Diamantina Institute, The University of Queensland, Brisbane, Queensland, 4072, Australia. ${ }^{7}$ Centre for Healthcare Improvement, Queensland Health, Brisbane, Queensland, 4029, Australia. ${ }^{8}$ School of Medicine, The University of Queensland, Brisbane, Queensland, 4072, Australia. ${ }^{9}$ Department of Endocrinology, Metro North Hospital \& Health Service, Queensland Health, Brisbane, Queensland, 4029, Australia.

Published: 31 May 2013

\section{doi:10.1186/1757-1146-6-S1-P8}

Cite this article as: Lazzarini et al:: Queensland diabetic foot hospitalisations (2005-10): in what state is our foot hospital problem? Journal of Foot and Ankle Research 2013 6(Suppl 1):P8.

* Correspondence: Peter_Lazzarini@health.qld.gov.au

${ }^{1}$ Allied Health Research Collaborative, Metro North Hospital \& Health Service,

Queensland Health, Brisbane, Queensland, 4032, Australia

Full list of author information is available at the end of the article 